\title{
Application of a Disposable Screen-Printed Electrode to Depression Diagnosis for Laboratory Rats Based on Blood Serotonin Detection
}

\author{
Min LıU,* Jiajie Hu,** Tian MA,** Shi WANG,** and Hong Ding**广 \\ *Department of Pharmacy, South-Central University for Nationalities, Wuhan 430074, P. R. China \\ **Department of Pharmacy, Wuhan University, Wuhan 430072, P. R. China
}

\begin{abstract}
No exact and digital diagnostic methods for depression have been found. In this study, we prepared a 5-HT biosensor based on screen-printed electrode and applied it to a rat depression model caused by chronic unpredictable mild stress (CUMS). During CUMS, the blood 5-HT and the depression behavior of the depressed rats and the rats treated by antidepressants were recorded. The correlation coefficient of 5-HT was $0.9966\left(0-4 \times 10^{-6} \mathrm{M}\right)$ on the sensor. Results demonstrated that 5-HT level of the depressed rats declined while the depression behavior was aggravated. Fluoxetine $(20 \mathrm{mg} / \mathrm{kg})$ and lentinan $(10,20 \mathrm{mg} / \mathrm{kg})$ mildly elevated 5-HT level and slowly regulated the behavior. Mifepristone $(20 \mathrm{mg} / \mathrm{kg})$ and Rhizoma Coptidis water extract $(10,20,100 \mathrm{mg} / \mathrm{kg})$ quickly reversed 5-HT level and the depression behavior. This sensor can accurately test blood 5-HT and might be applied to rapid diagnosis for depression and evaluation of antidepressants effect.
\end{abstract}

(Received March 17, 2011; Accepted June 14, 2011; Published August 10, 2011)

\section{Introduction}

Depression is one of the most common psychiatric diseases; it exerts devastating impairment on quality of life due to its high morbidity and recurrence rate. Misdiagnosis derived from ambiguous depression symptom criteria in depression scales makes it significant to develop a new and reliable diagnostic method. Serotonin (5-HT) level in plasma turns out to be a promising marker of depression, due to its ability of revealing the depressive level. ${ }^{1,2}$ Thus, fast detection of blood 5-HT is significant to the diagnosis and prognosis of the depressed patients.

Approaches like HPLC-ECD and ELISA have been widely used to determine 5-HT level. ${ }^{3,4}$ However, these methods are cumbersome and time-consuming, which makes them unsuitable for clinical rapid detection. HPLC-ECD combined with micro-dialysis is generally used for brain 5-HT test but is unsuitable for blood 5-HT determination., ${ }^{5,6}$ Voltammetric sensors for the 5-HT detection in biosamples have been widely used during the last few years: ${ }^{7,8}$ glassy carbon electrode which possesses high sensitivity and selectivity has been invented to test blood 5-HT, but the reaction surface of the electrode needs to be remade after each test, which results in limited application for pharmacological study. ${ }^{9}$ Recently, screen-printed electrode has attracted an increasing interest because of the various advantages including simple fabrication, low-cost, small size, disposability and ease of mass prodution. ${ }^{10,11}$

We prepared a 5-HT biosensor based on a Nafion membrane-coated colloidal gold screen-printed electrode

$\dagger$ To whom correspondence should be addressed.

E-mail: dinghongpharmacy05@tom.com
(Nafion/CGSPE) and applied it to mice brain and blood 5-HT test. A positive correlationship between brain and blood 5-HT level of mice was found. ${ }^{12}$ However, whether blood 5-HT can reflect the depression level has been disputed. Some regard that the depression level has negative correlation with blood 5-HT concentration while others maintain the opposite. ${ }^{13,14}$ Moreover, treatment of antidepressants changes the blood 5-HT level significantly. The blood 5-HT level after 1 day of fluoxetine (one of the typical selective serotonin reuptake inhibitors (SSRIs)) treatment is correlated to the depression degree $(P<0.05) .{ }^{15}$ The treatment of Paroxetine (SSRIs) also has the similar effect. ${ }^{16}$

In this study, we built a chronic unpredictable mild stress model (CUMS) on rats which mimicked the depression of people and tried to find the relationship between depression behavior and blood 5-HT level. During the 7-week CUMS, we recorded their depression activities and determined the rats' blood 5-HT level by our sensor. In addition, different antidepressants were used. Fluoxetine (FLU) is a typical antidepressant for clinical use. Mifepristone (MIF) affects the hypothalamic-pituitary-adrenal axis which regulates blood cortisol level to further influence 5-HT level in depression patients. ${ }^{17,18}$ Lentinan (LNT) increases the long-term potentiation of the rat in dentate gyrus and regulates the immunologic factors that affect the depression behavior significantly. ${ }^{19,20}$ Berberine extracted from Rhizoma Coptidis may have latent antidepressant effect on rodents. However, its poor water-solubility limited the application to clinic use. In this study, we administrated Rhizoma Coptidis water extract (BER) to the rats instead. ${ }^{21,22}$ After that, we investigated the correlation between blood 5-HT and behavior changes of the rats treated by these antidepressants. ${ }^{23}$ 


\section{Experimental}

\section{Reagents and chemicals}

5-Hydroxytryptamine sulfate, dopamine hydrochloride (DA), uric acid (UA) and ascorbic acid (AA) were purchased from Sigma (USA). The $0.1 \mathrm{M}$ phosphate buffer solution (PBS) at pH 7.4 was prepared as supporting electrolyte. Colloidal gold solution and $1 \%(\mathrm{w} / \mathrm{v})$ solution of Nafion was supplied by the Department of Pharmacy of Wuhan University. ${ }^{24}$

\section{Apparatus}

Electrochemical experiments such as cyclic voltmmetry and differential pulse voltammetry (DPV) were performed using a CHI800C electrochemical workstation (Shanghai Chenhua Instrument Ltd., China). The operating conditions to record differential pulse voltammograms were: pulse amplitude, $50 \mathrm{mV}$; sample width, $20 \mathrm{~ms}$; pulse width, $20 \mathrm{~ms}$; pulse period, $200 \mathrm{~ms}$; scan rate, $20 \mathrm{mV} / \mathrm{s}$; sensitivity, $100 \mathrm{nA} / \mathrm{V}$. The blood was treated by Ultrasonic Processor (BILON 98-III, Shanghai, China)

\section{Preparation of the modified electrode}

The electrodes were prepared using the screen-printed technique. Colloidal gold-modified graphite ink was included in the counter electrode and the working electrode. Nafion $(2.0 \mu \mathrm{L}, 1 \%)$ added to the surface of the SPEs was evaporated at room temperature. ${ }^{12}$

\section{Preparation of drugs}

Fluoxetine (Eli Lilly, USA), mifepristone (Beijing Zizhu Pharm. Ltd., China), lentinan (Wuhan Di'ao Pharm. Ltd., China) and Rhizoma Coptidis water extract (Xi' an Tongjiang Bio. Ltd., China) were each suspended by doubly distilled water.

\section{Animals grouping and administration}

One hundred and eight male Sprague-Dawley rats (180 - 200 g, Wuhan University Laboratory Animal Center) were normally fed for 1 week. ${ }^{25}$ Room temperature, $18-22^{\circ} \mathrm{C} ; 12 / 12 \mathrm{~h}$ day and night alternation; sufficient water and food supply. All animals handling procedures were performed in accordance with the guidelines published in the European Communities Council Directive of November 24, 1986 (86/609/EEC) and the P. R. China legislation for the use and care of laboratory animals. After 1 week of $1 \%$ sucrose solution adaptation, no food or water was supplied for $23 \mathrm{~h}$, and the intake of tap water and $1 \%$ sucrose of each rat in $1 \mathrm{~h}$ was measured. Based on the approximate intake of $1 \%$ sucrose, the rats were divided into nine groups: control group, model group, FLU group, MIF group, LNT (10, $20 \mathrm{mg} / \mathrm{kg})$ groups, BER $(10,20,100 \mathrm{mg} / \mathrm{kg})$ groups. Except for the control group, the other eight groups received CUMS for seven weeks (week 1 - 7). ${ }^{26}$ The CUMS procedure (eight stressors) was as follows: Food or water deprivation $(23 \mathrm{~h})$; cage tilt $\left(45^{\circ}, 23 \mathrm{~h}\right)$; continuous overnight illumination; soiled cage $(100 \mathrm{ml}$ of water spilled onto the bedding $(23 \mathrm{~h})$; cold water swimming $\left(4^{\circ} \mathrm{C}\right.$ for $\left.5 \mathrm{~min}\right)$; empty water bottles $(23 \mathrm{~h}) ; 2 \mathrm{~h}$ behavior restraint in a tube (diameter, $8 \mathrm{~cm}$; length, $20 \mathrm{~cm}$ ); intermittent illumination (light on and off every $2 \mathrm{~h}$ ). These stressors lasted for 7 weeks and each animal received one stress per day individually. ${ }^{27}$ After four weeks, sucrose intake of the rats under CUMS was obviously lower than that of control group. Then, from week 5 to 7, FLU group received $20 \mathrm{mg} / \mathrm{kg}$ fluoxetine orally; MIF group received $20 \mathrm{mg} / \mathrm{kg}$ mifepristone orally. ${ }^{23}$ LNT groups received oral
$10,20 \mathrm{mg} / \mathrm{kg}$ lentinan individually; ${ }^{28}$ BER groups received oral 10, 20, $100 \mathrm{mg} / \mathrm{kg}$ Rhizoma Coptidis water extract. The control group and model group received physiological saline. During week $1-7$, the sucrose preference, locomotor activity and blood 5-HT level of the rats were recorded.

The platelet-rich plasma (PRP) with EDTA as anticoagulant from each rat was obtained by centrifugation $(935 \mathrm{~g})$ for $70 \mathrm{~s}$ at room temperature. The upper liquids were destroyed by sonication $\left(20 \mathrm{kHz}\right.$, amplitude $8 \times 10^{-3} \mathrm{~mm}$ for $\left.30 \mathrm{~s}\right)$ and denatured by adding $0.5 \mathrm{M} \mathrm{HClO}_{4}$. The blood samples were unstable, so they were taken for analysis immediately in duplicate. ${ }^{29}$

\section{Sucrose preference test and measurement of locomotor activity}

The sucrose preference test was employed to define anhedonia, which involved $23 \mathrm{~h}$ of food and water deprivation, followed by presentation of $1 \%$ sucrose solution and tap water for 1 h. ${ }^{30,31}$ Intake was measured by measuring the volume of sucrose solution and water. The sucrose preference test was repeated every week and carried out in the home cage to minimize extraneous novelty and disturbance. The sucrose preference (SP) was calculated according to the following ratio: $\mathrm{SP}=$ sucrose intake/(sucrose intake + water intake) $\times 100 \%$.

The locomotor activity of rats was measured by an activity-monitoring system based on a previous report with modification. ${ }^{32,33}$ The apparatus consists of a metallic box $(75 \times 75 \times 50 \mathrm{~cm})$ and the bottom was painted in $5 \times 5$ squares. The rats were placed in the center and their behavior was noted immediately; observations continued for $5 \mathrm{~min}$. The parameters such as square number (movement distance) and rearing number were recorded by video camera and registered in the computer.

\section{Statistical analysis}

The Excel and SPSS 13.0 software were used to calculate means and standard deviations. ANOVA or t-test was used to analyze weight, $1 \%$ sucrose intake, locomotor activity and 5-HT level. The level of significance was set at $P<0.05$.

\section{Results}

\section{Real sample analysis}

$\mathrm{CV}$ behavior of 5-HT on Nafion/CGSPE showed great sensitivity. The calibration curves obtained by DPV indicated that 5-HT can be safely estimated in physiological concentration range. ${ }^{12}$ DA, AA and UA over the physiological level did not interfere with the test of 5-HT, which demonstrates high selectivity. ${ }^{12}$

Fixed 5-HT concentrations of $0.1,1.0$, and $10.0 \mu \mathrm{M}$ were tested by our Nafion/CGSPE; the relative standard deviations of the modified electrode were respectively 7.21, 5.33, and 8.49, which indicated a high degree of accuracy of the Nafion/CGSPE.

The Nafion/CGSPE was thus applied for the detection of 5-HT in rat PRP using the DPV mode. With exogenous 5-HT spiking $(0.05,0.1,0.2,0.5,1.0 \mu \mathrm{M})$, the catalytic peak current $\left(i_{\mathrm{p}}\right)$ was found to rise with the increase of the concentration of 5-HT (Fig. 1, a-f). The graph plotted between $i_{\mathrm{p}}$ and 5-HT concentration $(C)$ can be represented by the equation $i_{\mathrm{p}}(\mu \mathrm{A})=$ $0.1495 C(\mu \mathrm{M})+0.0598$; the correlation coefficient is 0.9983 . The limit of detection was $10.0 \mathrm{nM}$. This sensitivity was then compared with the previous result without blood components: $i_{\mathrm{p}}(\mu \mathrm{A})=0.095 C(\mu \mathrm{M})$ from 0.02 to $5 \mu \mathrm{M}$, with the limit of detection $5.0 \mathrm{nM} .^{12}$ The sensitivity was a little larger than the previous work, and the linearity of the range $(0.05,0.1,0.2,0.5$, $1.0 \mu \mathrm{M}$ ) could satisfy the 5-HT detection of the rats' experiment. 


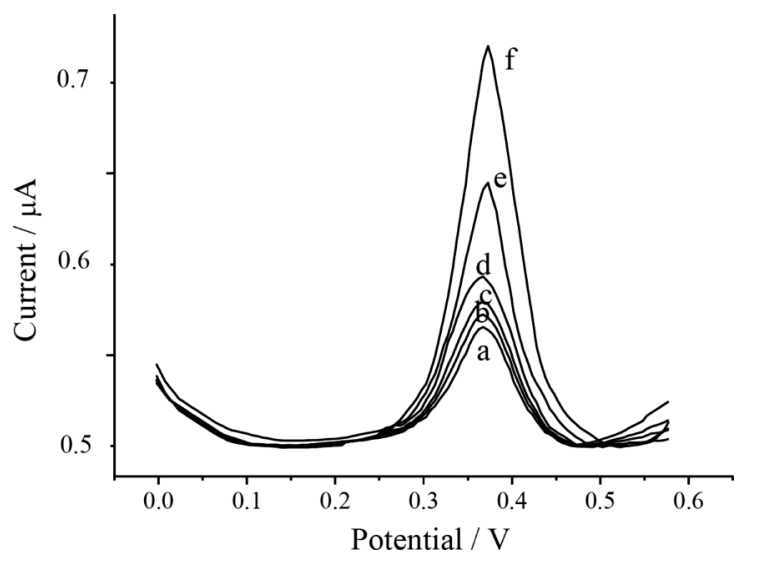

Fig. 1 DPVs for (a) 0, (b) 0.05, (c) 0.1 , (d) 0.2 , (e) 0.5 , (f) $1.0 \mu \mathrm{M}$ 5-HT in rat PRP.

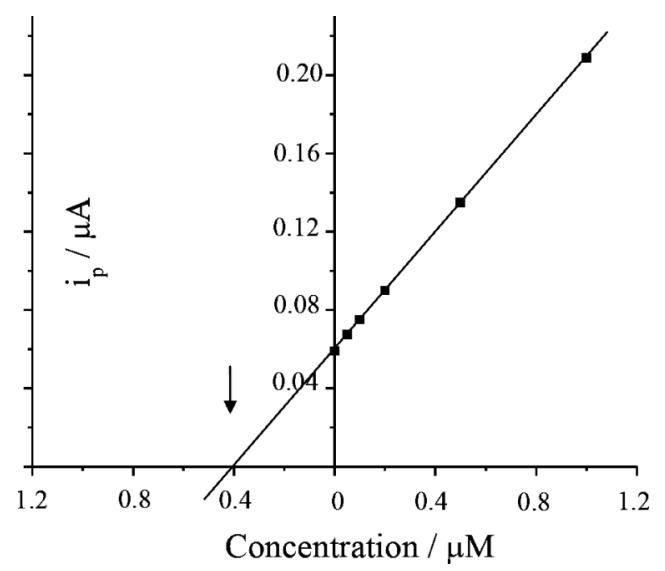

Fig. 2 The standard addition curve of 5-HT by adding $0,0.05,0.1$, $0.2,0.5$, or $1.0 \mu \mathrm{M} 5$-HT in rat PRP. The arrowhead showed the 5-HT level in rat PRP.

This indicates that this Nafion/CGSPE can be safely applied to rats PRP 5-HT test during the research of depression diagnosis. The calibration line is shown in Fig. 2; the 5-HT concentration in rat PRP was $0.4 \mu \mathrm{M}$ (Fig. 1, a).

\section{PRP 5-HT level of the rats during CUMS}

The PRP 5-HT level of the rats in control group fluctuated slightly between 0.3 and $0.5 \mu \mathrm{M}$. However, the 5-HT level of model rats gradually became lower, the declining rate increased obviously during the first three weeks. In week 3, 5-HT level of the depressed rats was $0.18 \mu \mathrm{m}$, and in week 6,5 -HT level of the depressed rats was only $0.09 \mu \mathrm{M}$ (Fig. 3). However, from week 5, MIF rapidly increased the PRP 5-HT level, but FLU elevated the PRP 5-HT level suddenly in the following week 6 (Fig. 4). LNT $(10,20 \mathrm{mg} / \mathrm{kg})$ increased the 5-HT level slowly. BER $(10,20,100 \mathrm{mg} / \mathrm{kg})$ steadily increased the 5-HT to normal level.

\section{Behavior of the rats during CUMS}

During the CUMS (week 1-7), the square and rearing number of the rats in the model group were lower than that of control group $(P<0.001)$. The square and rearing number of the rats in FLU group increased nearly to that of the control group $(P>0.05)$. MIF group was even better than the control

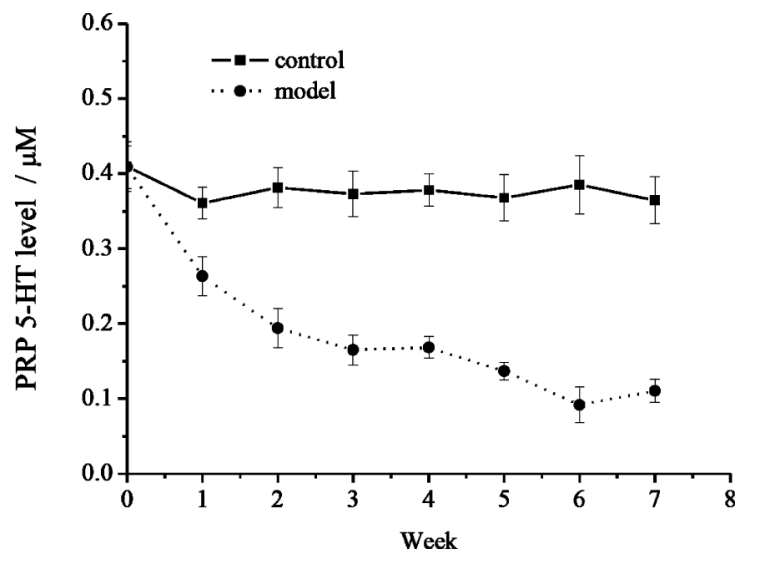

Fig. 3 PRP 5-HT level of the CUMS rats compared with the rats in the control group.
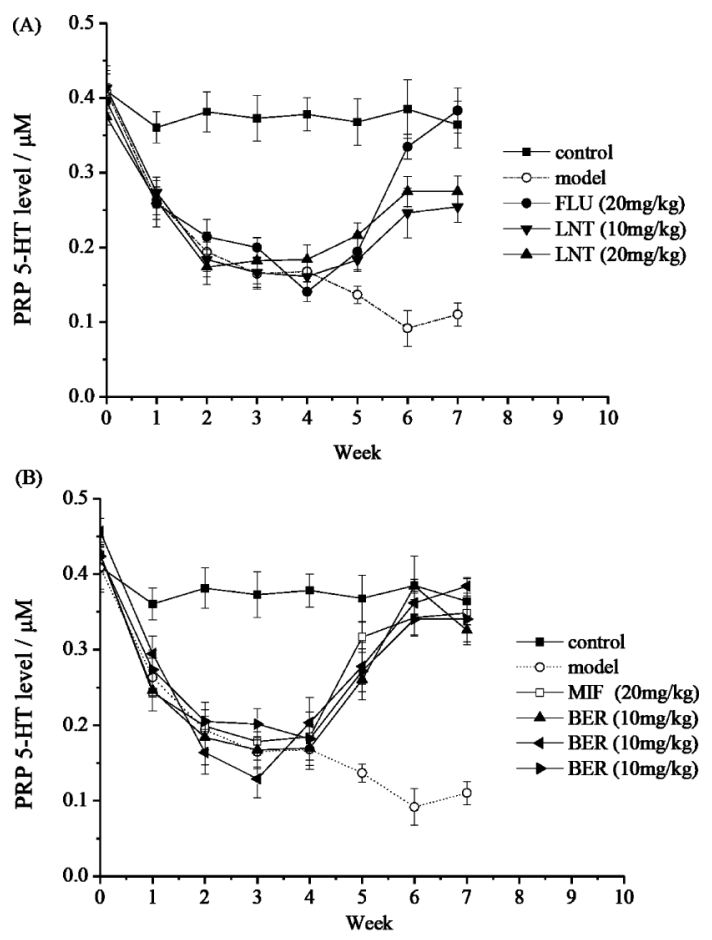

Fig. 4 PRP 5-HT level of the CUMS rats treated by antidepressants.

group. LNT (10, $20 \mathrm{mg} / \mathrm{kg})$ and $\operatorname{BER}(10,20,100 \mathrm{mg} / \mathrm{kg})$ increased the locomotor in both square and rearing number. The sucrose preference rate of the rats in model group declined greatly from the first week while it did not change in control group. After treated, the rate of the rats in MIF and LNT $(10,20 \mathrm{mg} / \mathrm{kg})$ group increased immediately one week after drug treatment. FLU effect was one week delayed but raised promptly (week 6). BER $(10,20,100 \mathrm{mg} / \mathrm{kg}$ ) increased the preference rate softly from week 5 and gained best in week 7 .

We investigated the correlationship between PRP 5-HT level and sucrose preference rate during CUMS to find if the PRP 5-HT could reflect the anhedonia of the rats. Figure 5(A) showed that the correlation coefficient was 0.65 , indicating a fine correlationship. At the same time, we studied the correlationship between PRP 5-HT level and locomotor actitivity to find if the PRP 5-HT can reflect the depression behavior of the rats. The correlation coefficient was 0.76 (Fig. 5(B)). Thus, 

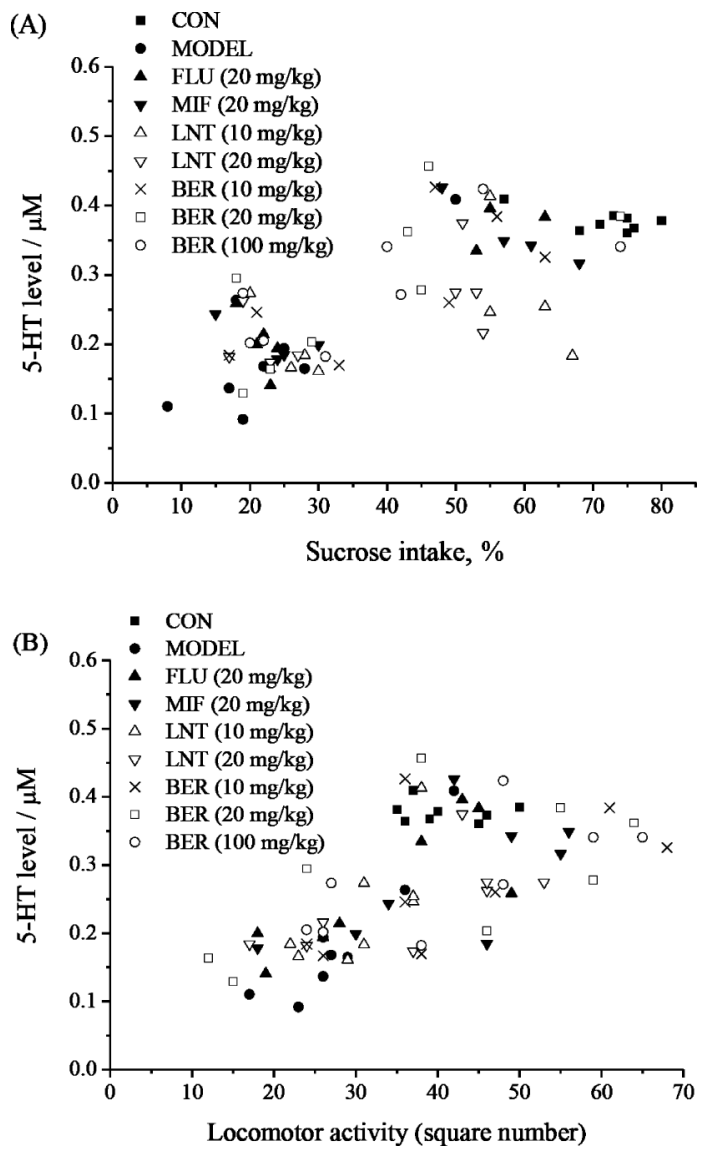

Fig. 5 (A) The correlationship of the sucrose intake and PRP 5-HT of the CUMS rats. (B) The correlationship of the locomotor and PRP 5-HT of the CUMS rats.

correlation between PRP 5-HT and locomotor activity is more evident than that between PRP 5-HT and sucrose preference.

\section{Discussion}

In this study, we prepared depression rats with CUMS and evaluated value the depression level with the sucrose preference, locomotor activity and NIH. Sucrose preference of the rats reflects "anhedonia"; locomotor activity is one of the most typical depression behaviors; NIH indicates the drive to eat and the fear of venturing into a novel environment. ${ }^{27}$ At the same time, we tested the PRP 5-HT with our sensor based on Nafion/CGSPE. During weeks 1-4, sucrose preference and locomotor activity of rats in model groups decreased gradually, while the values in the control group are unchanged which indicated that the depression model was prepared successfully. Meanwhile, the PRP 5-HT level of the depression rats also declined accordingly but that in the control group just changed slightly. These results suggested that PRP 5-HT level which showed us the depression level of rats could be considered a biological marker for depression diagnosis.

From weeks 5 to 7 , when the drugs were administrated to the rats, the sucrose preference, locomotor activity and PRP 5-HT level of each group recovered differently. FLU (20 mg/kg) can return the depression behaviors detected by CUMS to normal level, but the antidepressant effect were two weeks delayed. Interestingly, the PRP 5-HT elevated the same way as sucrose preference; the slack effective antidepressant was totally reflected. MIF $(20 \mathrm{mg} / \mathrm{kg})$ has the best curative effect on sucrose preference and the body weight of the rats recovered quickly. MIF lifts the PRP 5-HT level quickly, along with the effect on depression behavior. The antidepressant effect of BER $(10,20,100 \mathrm{mg} / \mathrm{kg})$ was quite similar to that of MIF, which might interfere in the glucocorticoid system. When BER $(10,20,100 \mathrm{mg} / \mathrm{kg})$ regulated the body weight and NIH of the rats, the depression behavior and PRP 5-HT level jumped up promptly and synchronously. PRP 5-HT shows great potential to be a biological marker of curative effect for fluoxetine, mifepristone and Rhizoma Coptidis water extract. Whether it can be applied to LNT remains unclear.

The Nafion/CGSPE in this study are suitable for 5-HT detection in biological samples, could be used to antidepressant screening and clinical diagnosis. According to treatment of the clinical antidepressant, PRP 5-HT shows great potential to be a biological marker of curative effect for fluoxetine, mifepristone and Rhizoma Coptidis water extract. Whether it can be applied to lentinan remains unclear. Variance of PRP 5-HT might be a result of neurotrophic effect of antidepressants.

\section{Acknowledgements}

We greatly thank Rongbin Zeng (Nanjing Tianding Institute of Biotechnology), for providing related supplies and experimental assistance.

\section{References}

1. I. Karakulova, Zh. Nevrol. Psikhiatr. Im. S. S. Korsakova, 2006, 106, 52 .

2. K. Wu, J. Fei, and S. Hu, Anal. Biochem., 2003, 318, 100.

3. T. N. Wise, L. M. Arnold, and V. Maletic, CNS Spectrums, 2005, $10,1$.

4. T. Yoshitake, J. Kehr, K. Todoroki, H. Nohta, and M. Yamaguchi, Biomed. Chromatogr., 2006, 20, 267.

5. N. Kato, T. Kojima, and S. Yoshiyagawa, J. Chromatogr., A, 2007, 1145, 229.

6. J. D. Cooper, K. E. Heppert, M. I. Davies, and S. M. Lunte, J. Neurosci. Methods, 2007, 160, 269.

7. R. N. Goyal, V. K. Gupta, M. Oyama, and N. Bachheti, Talanta, 2007, 72, 976.

8. H. Yao, S. G. Li, Y. H. Tang, Y. Chen, Y. Z. Chen, and X. H. Lin, Electrochim. Acta, 2009, 54, 4607.

9. K. Ueda, R. Yonemoto, K. Komagoe, K. Masuda, N. Hanioka, S. Narimatsu, and T. Katsu, Anal. Chim. Acta, 2006, 565,36 .

10. S. Miserere, S. Ledru, N. Ruille, S. Griveau, M. Boujtitab, and F. Bedioui, Electrochem. Commun., 2006, 8, 238.

11. G. Zhao, F. Xing, and S. Deng, Electrochem. Commun., 2007, 9, 1263.

12. M. Liu, J. Xiang, J. Zhou, and H. Ding, J. Electroanal. Chem., 2010, 640, 1.

13. N. D. Demchuk and A. A. Shutov, Zh. Nevrol. Psikhiatr. Im. S. S. Korsakova, 2008, 108, 29.

14. J. Roggenbach, B. M. Oerlinghausen, L. Franke, R. Uebelhack, S. Blank, and B. Ahrens, J. Neural Transm., 2007, 114, 479.

15. J. C. Alvarez, N. Gluck, A. Fallet, A. Grégoire, J. F. Chevalier, C. Advenier, and O. Spreux-Varoquaux, Psychopharmacology (Berlin, Ger.), 1999, 143, 97.

16. D. Mück-Šeler, N. Pivac, M. Šagud, M. Jakovljevic, 
and P. A. Mihaljevic, Progress Neuropsychopharm Biol. Psychiatry, 2002, 26, 1235.

17. J. C. Bernard and T. R. Robert, Neuropsychopharmacology, 2006, 31, 2793.

18. G. E. Tafet, V. P. Idoyaga-Vargas, D. P. Abulafia, J. M. Calandria, S. S. Roffman, A. Chiovetta, and M. Shinitzky, Cogn. Affect. Behav. Neurosci., 2001, 1, 388.

19. Y. Edagawa, M. Smriga, N. Nishiyama, and H. Saito, Neurosci. Lett., 2001, 314, 139.

20. V. Vetvicka and J. C. Yvin, Int. Immunopharmacol., 2004 4,721 .

21. W. H. Peng, K. L. Lo, Y. H. Lee, T. H. Hung, and Y. C. Lin, Life Sci., 2007, 81, 933

22. S. K. Kulkarni and A. Dhir, Eur. J. Pharm. Sci., 2008, 589 , 163.

23. A. Wesolowska and A. Nikiforuk, Eur. J. Pharm. Sci., 2008, 582,88 .

24. J. Turkevich, P. C. Stevenson, and J. Hillier, Discussions Faraday Soc., 1951, 11, 55.
25. J. Y. Tang, Y. S. Zeng, Q. G. Chen, Y. J. Qin, S. J. Chen, and Z. Q. Zhong, J. Sun Yat-sen Univ. (Med. Sci.), 2008, 29, 541.

26. P. Willner, Psychopharmacology, 1997, 134, 319.

27. H. X. Dang, Y. Chen, X. M. Liu, Q. Wang, L. Wang, W. Jia, and Y. Wang, Prog. Neuro-Psychoph., 2009, 33, 1417.

28. B. J. Carroll and R. T. Rubin, Neuropsychopharmacology, 2005, 31, 2793.

29. D. Mück-Šeler, N. Pivac, M. Mustapic, Z. Crncevic, M. Jakovljevic, and M. Sagud, Psychiat. Res., 2004, 127, 217.

30. P. Willner, A. Towell, D. Sampson, S. Sophokleous, and R. Muscat, Psychopharmacology, 1987, 93, 358.

31. S. Bekris, K. Antonious, S. Daskas, and Z. Papadopoulous-Daifoti, Behav. Brain Res., 2005, 161, 45.

32. N. M. Lind, M. Vinther, R. P. Hemmingsen, and A. K. Hansen, J. Neurosci. Methods, 2005, 143, 123.

33. W. L. Mai, Q. Wang, X. M. Liu, Y. H. Li, S. G. Chen, L. W. Wang, Y. F. Zhu, and Z. Q. Feng, Acta Lab Anim. Sci. Sin., 2008, 16, 570. 